\title{
Univalent harmonic mappings II
}

\author{
by Albert E. Livingston (Newark, Del.)
}

\begin{abstract}
Let $a<0<b$ and $\Omega(a, b)=\mathbb{C}-((-\infty, a] \cup[b,+\infty))$ and $U=\{z:|z|<1\}$. We consider the class $S_{H}(U, \Omega(a, b))$ of functions $f$ which are univalent, harmonic and sense-preserving with $f(U)=\Omega$ and satisfying $f(0)=0, f_{z}(0)>0$ and $f_{\bar{z}}(0)=0$.
\end{abstract}

1. Introduction. Let $S_{H}$ be the class of functions $f$ which are univalent, sense-preserving, harmonic mappings of the unit disk $U=\{z:|z|<1\}$ and satisfy $f(0)=0$ and $f_{z}(0)>0$. Let $F$ and $G$ be analytic in $U$ with $F(0)=G(0)=0$ and $\operatorname{Re} f(z)=\operatorname{Re} F(z)$ and $\operatorname{Im} f(z)=\operatorname{Re} G(z)$ for $z$ in $U$. Then $h=(F+i G) / 2$ and $g=(F-i G) / 2$ are analytic in $U$ and $f=h+\bar{g}$. $f$ is locally one-to-one and sense-preserving if and only if $\left|g^{\prime}(z)\right|<\left|h^{\prime}(z)\right|$ for $z$ in $U$ (cf. [4]). If $h(z)=a_{1} z+a_{2} z^{2}+\ldots, a_{1}>0$, and $g(z)=b_{1} z+b_{2} z^{2}+\ldots$ for $z$ in $U$, it follows that $\left|b_{1}\right|<a_{1}$ and hence $a_{1} f-\overline{b_{1} f}$ also belongs to $S_{H}$. Thus consideration is often restricted to the subclass $S_{H}^{0}$ of $S_{H}$ consisting of those functions in $S_{H}$ with $f_{\bar{z}}(0)=0$.

Various authors have studied subclasses of $S_{H}^{0}$ consisting of functions mapping $U$ onto a specific simply connected domain. See for example Hengartner and Schober [5], Abu-Muhanna and Schober [1], and Cima and the author [2], [3]. Recently the author [7] studied the subclass of $S_{H}^{0}$ consisting of functions mapping $U$ onto the plane with the interval $(-\infty, a], a<0$, removed. See also Hengartner and Schober [6]. In the present paper we consider the case when $f(U)$ is $C-((-\infty, a] \cup[b,+\infty)), a<0<b$.

Let $a<0<b$ and $\Omega(a, b)=\mathbb{C}-((-\infty, a] \cup[b,+\infty))$. Then $S_{H}(U, \Omega(a, b))$ is the class of functions $f$ in $S_{H}^{0}$ with $f(U)=\Omega(a, b)$. Without loss of generality, we assume that $a+b \geq 0$.

In the sequel $F$ and $G$ will be functions analytic in $U$ with $F(0)=G(0)=$ $0, \operatorname{Re} f(z)=\operatorname{Re} F(z)$ and $\operatorname{Im} f(z)=\operatorname{Re} G(z)$ for $z$ in $U$. If $h=(F+i G) / 2$ and $g=(F-i G) / 2$, then $f=h+\bar{g}$ and $\left|g^{\prime}(z)\right|<\left|h^{\prime}(z)\right|$ for $z$ in $U$.

1991 Mathematics Subject Classification: 30C55, 31A05.

Key words and phrases: univalent harmonic mappings, coefficient bounds, distortion theorems. 
2. Preliminary lemmas. Let $\mathcal{P}$ be the class of functions $P(z)$ which are analytic in $U$ with $P(0)=1$ and $\operatorname{Re} P(z)>0$ for $z$ in $U$. To get an integral representation of functions in $S_{H}(U, \Omega(a, b))$ we require a few lemmas.

LEMma 1. Let

$$
T(x)=\int_{0}^{1}\left(\frac{a(1+t)^{2}}{\left(1+x t+t^{2}\right)^{2}}+\frac{b(1-t)^{2}}{\left(1-x t+t^{2}\right)^{2}}\right) d t
$$

and

$$
S(x)=\int_{0}^{1}\left(\frac{a(1-t)^{2}}{\left(1+x t+t^{2}\right)^{2}}+\frac{b(1+t)^{2}}{\left(1-x t+t^{2}\right)^{2}}\right) d t
$$

where $a<0<b, a+b \geq 0$ and $-2<x<2$. There exist unique numbers $c_{1}$ and $c_{2}$ with $-2<c_{1}<0<c_{2}<2$ so that $S\left(c_{1}\right)=T\left(c_{2}\right)=0$. Moreover, $T(x) \leq 0 \leq S(x)$ if and only if $c_{1} \leq x \leq c_{2}$.

Proof. We note that

$$
S(x)-T(x)=\int_{0}^{1}\left(\frac{-2 a t}{\left(1+x t+t^{2}\right)^{2}}+\frac{2 b t}{\left(1-x t+t^{2}\right)^{2}}\right) d t \geq 0 .
$$

Thus $T(x) \leq S(x)$ for $-2<x<2$. Also, it is easily checked that $T^{\prime}(x)>0$ and $S^{\prime}(x)>0$ for $-2<x<2$. Thus $T(x)$ and $S(x)$ are both strictly increasing. Since $\lim _{x \rightarrow-2} T(x)=\lim _{x \rightarrow-2} S(x)=-\infty$ and $\lim _{x \rightarrow 2} T(x)=$ $\lim _{x \rightarrow 2} S(x)=+\infty$, it follows that there exist unique $c_{1}$ and $c_{2}$ so that $S\left(c_{1}\right)=T\left(c_{2}\right)=0$ and that $c_{1}<c_{2}$. Moreover, $S(0)>0$, thus $c_{1}<0$ and $T(x) \leq 0 \leq S(x)$ if and only if $c_{1} \leq x \leq c_{2}$.

Lemma 2. Let $P(z)$ be in $\mathcal{P}$ and

$$
\begin{aligned}
Q(x)= & a \int_{0}^{1} \frac{1-t^{2}}{\left(1+x t+t^{2}\right)^{2}} \operatorname{Re} P(t) d t \\
& +b \int_{0}^{1} \frac{1-t^{2}}{\left(1-x t+t^{2}\right)^{2}} \operatorname{Re} P(-t) d t
\end{aligned}
$$

where $a<0<b, a+b \geq 0$ and $-2<x<2$. There exists a unique $c,-2<c<2$, so that $Q(c)=0$.

P r o of. It is easily checked that $Q^{\prime}(x)>0$ for $-2<x<2, \lim _{x \rightarrow-2} Q(x)$ $=-\infty$ and $\lim _{x \rightarrow 2} Q(x)=+\infty$. The lemma then follows.

LEMMA 3. With the same hypotheses as in Lemma 2 and with $a$ and $b$ fixed we have $c_{1} \leq c \leq c_{2}$ where $c_{1}$ and $c_{2}$ are given in Lemma 1 . The range for $c$ is sharp in the sense that for each $c, c_{1} \leq c \leq c_{2}$, there exists $P(z)$ in $\mathcal{P}$ such that the corresponding $Q$ given by (2.3) satisfies $Q(c)=0$. 
Proof. Let $P(z)$ be in $\mathcal{P}$ and the corresponding $Q$ in (2.3) satisfy $Q(c)$ $=0$. Using the inequalities $(1-|z|) /(1+|z|) \leq \operatorname{Re} P(z) \leq(1+|z|) /(1-|z|)$ for $z$ in $U$, we obtain

$$
\begin{aligned}
& \frac{(1-t)^{2}}{\left(1+c t+t^{2}\right)^{2}} \leq \frac{\left(1-t^{2}\right) \operatorname{Re} P(t)}{\left(1+c t+t^{2}\right)^{2}} \leq \frac{(1+t)^{2}}{\left(1+c t+t^{2}\right)^{2}}, \\
& \frac{(1-t)^{2}}{\left(1-c t+t^{2}\right)^{2}} \leq \frac{\left(1-t^{2}\right) \operatorname{Re} P(-t)}{\left(1-c t+t^{2}\right)^{2}} \leq \frac{(1+t)^{2}}{\left(1-c t+t^{2}\right)^{2}} .
\end{aligned}
$$

Since $a<0<b$, this gives

$$
\begin{aligned}
\int_{0}^{1}\left(\frac{a(1+t)^{2}}{\left(1+c t+t^{2}\right)^{2}}+\right. & \left.\frac{b(1-t)^{2}}{\left(1-c t+t^{2}\right)^{2}}\right) d t \\
& \leq Q(c) \leq \int_{0}^{1}\left(\frac{a(1-t)^{2}}{\left(1+c t+t^{2}\right)^{2}}+\frac{b(1+t)^{2}}{\left(1-c t+t^{2}\right)^{2}}\right) d t .
\end{aligned}
$$

Thus $T(c) \leq 0 \leq S(c)$ where $T$ and $S$ are given in Lemma 1. From Lemma 2 we have $c_{1} \leq c \leq c_{2}$.

To see that the range of $c$ is sharp, we note that $Q\left(c_{1}\right)=0$ when $P(z)=$ $(1-z) /(1+z)$ and $Q\left(c_{2}\right)=0$ when $P(z)=(1+z) /(1-z)$. If $c_{1}<c<c_{2}$ then $T(c)<0<S(c)$. That is,

$$
\begin{aligned}
\int_{0}^{1}\left(\frac{a(1+t)^{2}}{\left(1+c t+t^{2}\right)^{2}}+\right. & \left.\frac{b\left(1-t^{2}\right)}{\left(1-c t+t^{2}\right)^{2}}\right) d t \\
& <0<\int_{0}^{1}\left(\frac{a(1-t)^{2}}{\left(1+c t+t^{2}\right)^{2}}+\frac{b(1+t)^{2}}{\left(1-c t+t^{2}\right)^{2}}\right) d t
\end{aligned}
$$

With $c$ fixed, let

$$
\phi(P)=a \int_{0}^{1} \frac{\left(1-t^{2}\right) \operatorname{Re} P(t)}{\left(1+c t+t^{2}\right)^{2}} d t+b \int_{0}^{1} \frac{\left(1-t^{2}\right) \operatorname{Re} P(-t)}{\left(1-c t+t^{2}\right)^{2}} d t ;
$$

then $\phi$ is a real-valued continuous functional on the convex space $\mathcal{P}$. From (2.4) it follows that

$$
\phi\left(\frac{1+z}{1-z}\right)<0<\phi\left(\frac{1-z}{1+z}\right)
$$

For $0 \leq \lambda \leq 1$,

$$
\phi\left(\lambda \frac{1-z}{1+z}+(1-\lambda) \frac{1+z}{1-z}\right)
$$

is a real-valued continuous function of $\lambda$ for $0 \leq \lambda \leq 1$, with $\phi(0)<0<\phi(1)$. Then there is $\lambda_{1}$ so that $\phi\left(\lambda_{1}\right)=0$. The function $P_{1}(z)=\lambda_{1}(1-z) /(1+z)$ $+\left(1-\lambda_{1}\right)(1+z) /(1-z)$ is a member of $\mathcal{P}$ and the corresponding $Q$ defined by $(2.3)$ satisfies $Q(c)=0$. 
3. The class $S_{H}(U, \Omega(a, b))$. In the sequel the numbers $c, c_{1}$ and $c_{2}$ are those given by Lemmas $1-3$.

Let $\mathcal{F}(a, b)$ be the class of functions which have the form

$$
f(z)=A\left[\operatorname{Re} \int_{0}^{z} \frac{\left(1-\zeta^{2}\right) P(\zeta)}{2\left(1+c \zeta+\zeta^{2}\right)^{2}} d \zeta+i \operatorname{Im} \frac{z}{\left(1+c z+z^{2}\right)^{2}}\right]
$$

where

$$
A=b / \int_{0}^{1} \frac{\left(1-t^{2}\right) \operatorname{Re} P(t)}{\left(1+c t+t^{2}\right)^{2}} d t
$$

with $P(z)$ in $\mathcal{P}$ and $c$ is chosen so that $c_{1} \leq c \leq c_{2}$ and

$$
b / \int_{0}^{1} \frac{\left(1-t^{2}\right) \operatorname{Re} P(t)}{\left(1+c t+t^{2}\right)^{2}} d t=a / \int_{0}^{-1} \frac{\left(1-t^{2}\right) \operatorname{Re} P(t)}{\left(1+c t+t^{2}\right)^{2}} d t .
$$

We note that by Lemmas $1-3$, for each $P$ in $\mathcal{P}$ there is a unique $c, c_{1} \leq$ $c \leq c_{2}$, for which (3.2) is satisfied.

THEOREM 1. If $f$ is a member of $\mathcal{F}(a, b)$, then $f$ is harmonic, sensepreserving and univalent in $U$. Moreover, $f(U)$ is convex in the direction of the real axis and $f(U) \subset \Omega(a, b)$.

Proof. Let $f=h+\bar{g}=\operatorname{Re} F+i \operatorname{Re} G$; then

$$
F(z)=A \int_{0}^{z} \frac{(1-\zeta)^{2} P(\zeta)}{\left(1+c \zeta+\zeta^{2}\right)^{2}} d \zeta \quad \text { and } \quad G(z)=\frac{-i A z}{1+c z+t^{2}}
$$

Since

$$
\frac{g^{\prime}(z)}{h^{\prime}(z)}=\frac{F^{\prime}(z)-i G^{\prime}(z)}{F^{\prime}(z)+i G^{\prime}(z)}=\frac{P(z)-1}{P(z)+1},
$$

it follows that $\left|g^{\prime}(z)\right|<\left|h^{\prime}(z)\right|$ for $z$ in $U$. Thus $f$ is locally one-to-one and sense preserving in $U$.

Also,

$$
h(z)-g(z)=i G(z)=\frac{A z}{1+c z+z^{2}}
$$

maps $U$ onto a domain which is convex in the direction of the real axis. By a theorem of Clunie and Sheil-Small [4], $f$ is univalent and $f(U)$ is convex in the direction of the real axis. Also, $f(z)$ is real if and only if $z$ is real. Since $A>0$ and $\operatorname{Re} P(z)>0$, it follows that $f(r)=\operatorname{Re} F(r)$ is increasing in $[-1,1]$ and by $(3.2), \lim _{r \rightarrow-1^{+}} f(r)=a$ and $\lim _{r \rightarrow 1^{-}} f(r)=b$. Thus $f(U)$ omits $(-\infty, a]$ and $[b,+\infty)$. Hence $f(U) \subset \Omega(a, b)$.

Theorem 2. $S_{H}(U, \Omega(a, b)) \subset \mathcal{F}(a, b)$.

Proof. Let $f$ be a member of $S_{H}(U, \Omega(a, b))$ and $f=h+\bar{g}$. Since $\Omega(a, b)$ is convex in the direction of the real axis, by a result of Clunie and 
Sheil-Small [4], $h-g=i G$ is univalent and convex in the direction of the real axis. Thus $G$ is convex in the direction of the imaginary axis.

Let $h(z)=a_{1} z+a_{2} z^{2}+\ldots, a_{1}>0$, and $g(z)=b_{2} z^{2}+b_{3} z^{3}+\ldots ;$ then $G=-i(h-g)=-a_{1} i z+\ldots$ Since $f(U)=\Omega(a, b)$, it follows that $\operatorname{Re} G(z)=$ $\operatorname{Im} f(z)$ is 0 on the boundary of $U$. Since $G$ is convex in the direction of the imaginary axis, it follows that $G(U)$ is $\mathbb{C}$ slit along one or two infinite rays along the imaginary axis. Thus $G(z) /\left(-a_{1} i\right)$ maps $U$ into $\mathbb{C}$ slit along one or two infinite rays along the real axis. However, $G(z) /\left(-a_{1} i\right)$ is a member of the class $S$ of functions $q(z)$ analytic and univalent in $U$ and normalized by $q(0)=q^{\prime}(0)-1=0$. Making use of subordination arguments, it follows that $G(z) /\left(-a_{1} i\right)=z /\left(1+c z+z^{2}\right),-2 \leq c \leq 2$. Hence, $\operatorname{Im} f(r)=\operatorname{Re} G(r)=0$ for $-1<r<1$. Since $f$ is one-to-one and $f_{z}(0)>0$, the function $f(r)$ is increasing on $(-1,1)$. Thus $\lim _{r \rightarrow-1^{+}} f(r)=a$ and $\lim _{r \rightarrow 1^{-}} f(r)=b$.

Since $\left|g^{\prime}(z) / h^{\prime}(z)\right|<1$, it follows that

$$
P(z)=\left(h^{\prime}(z)+g^{\prime}(z)\right) /\left(h^{\prime}(z)-g^{\prime}(z)\right)
$$

is in $\mathcal{P}$. Thus, $h^{\prime}(z)+g^{\prime}(z)=\left(h^{\prime}(z)-g^{\prime}(z)\right) P(z)=i G^{\prime}(z) P(z)$.

Hence,

$$
F(z)=h(z)+g(z)=\int_{0}^{z} i G^{\prime}(\zeta) P(\zeta) d \zeta=a_{1} \int_{0}^{z} \frac{\left(1-\zeta^{2}\right) P(\zeta)}{\left(1+c \zeta+\zeta^{2}\right)^{2}} d \zeta .
$$

Therefore,

$$
f(z)=a_{1}\left[\operatorname{Re} \int_{0}^{z} \frac{\left(1-\zeta^{2}\right) P(\zeta)}{\left(1+c \zeta+\zeta^{2}\right)^{2}} d \zeta+i \operatorname{Im} \frac{z}{1+c z+z^{2}}\right]
$$

for some $c,-2 \leq c \leq 1$.

Since $a=\lim _{r \rightarrow-1^{+}} f(r)$ and $b=\lim _{r \rightarrow 1^{-}} f(r)$, we have

$$
a_{1} \int_{0}^{-1} \frac{\left(1-t^{2}\right) \operatorname{Re} P(t)}{\left(1+c t+t^{2}\right)^{2}} d t=a \quad \text { and } \quad a_{1} \int_{0}^{1} \frac{\left(1-t^{2}\right) \operatorname{Re} P(t)}{\left(1+c t+t^{2}\right)^{2}} d t=b .
$$

Thus $c$ must be such that

$$
a \int_{0}^{1} \frac{\left(1-t^{2}\right) \operatorname{Re} P(t)}{\left(1+c t+t^{2}\right)^{2}} d t+b \int_{0}^{1} \frac{\left(1-t^{2}\right) \operatorname{Re} P(-t)}{\left(1-c t+t^{2}\right)^{2}} d t=0 .
$$

By Lemmas 2 and 3 there is a unique $c, c_{1} \leq c \leq c_{2}$, satisfying (3.3). Thus $f$ is a member of $\mathcal{F}(a, b)$.

Lemma 4. $\mathcal{F}(a, b)$ is closed.

Pr o of. Let $f_{n}$ be a sequence in $\mathcal{F}(a, b)$ with $f_{n}$ converging to $f$ uniformly 
on compact subsets of $U$. Suppose

$$
\begin{aligned}
f_{n}(z)= & b\left(\int_{0}^{1} \frac{(1-t)^{2} \operatorname{Re} P_{n}(t)}{\left(1+d_{n} t+t^{2}\right)^{2}} d t\right)^{-1} \\
& \times\left[\operatorname{Re} \int_{0}^{z} \frac{\left(1-\zeta^{2}\right) P_{n}(\zeta)}{\left(1+d_{n} \zeta+\zeta^{2}\right)^{2}} d \zeta+i \operatorname{Im} \frac{z}{1+d_{n} z+z^{2}}\right],
\end{aligned}
$$

where $P_{n}$ is in $\mathcal{P}$ and $d_{n}$ satisfies (3.2) with $c_{1} \leq d_{n} \leq c_{2}$. Since $\mathcal{P}$ is normal and $c_{1} \leq d_{n} \leq c_{2}$ we may assume that $P_{n}$ converges uniformly on compact subsets of $U$ to $P(z)$ in $\mathcal{P}$ and $d_{n}$ converges to some $c$. It follows that (3.2) is satisfied for this $c$ and $P(z)$ and that $f$ has the form (3.1) and hence is a member of $\mathcal{F}(a, b)$.

Theorem 3. $\overline{S_{H}(U, \Omega(a, b))}=\mathcal{F}(a, b)$.

Proof. Let $f(z)$ have the form (3.1) where (3.2) is satisfied and let $r_{n}$ be a sequence with $0<r_{n}<1$ and $\lim r_{n}=1$. Let $P_{n}(z)=P\left(r_{n} z\right)$ and denote by $f_{n}(z)$ the function obtained from (3.1) and (3.2) by replacing $P(z)$ with $P_{n}(z)$. Let $c_{n}$ be the value of $c$ satisfying (3.2) when $P$ is replaced by $P_{n}$. We claim that $f_{n}$ is a member of $S_{H}(U, \Omega(a, b))$. To see this let

$$
A_{n}=b / \operatorname{Re} \int_{0}^{1} \frac{\left(1-\zeta^{2}\right) P_{n}(\zeta)}{\left(1+c_{n} \zeta+\zeta^{2}\right)^{2}} d \zeta, \quad F_{n}(z)=A_{n} \int_{0}^{z} \frac{\left(1-\zeta^{2}\right) P_{n}(\zeta)}{\left(1+c_{n} \zeta+\zeta^{2}\right)^{2}} d \zeta
$$

Let $s_{n}=\left[-c_{n}+i \sqrt{4-c_{n}^{2}}\right] / 2$; then $\left(1+c_{n} \zeta+\zeta^{2}\right)=\left(\zeta-s_{n}\right)\left(\zeta-\bar{s}_{n}\right)$. Since $P_{n}$ is analytic for $|z| \leq 1$, there exists $\delta>0$ so that for $\left|z-s_{n}\right|<\delta$,

$$
P_{n}(z)=P_{n}\left(s_{n}\right)+P_{n}^{\prime}\left(s_{n}\right)\left(z-s_{n}\right)+\frac{P_{n}^{\prime \prime}\left(s_{n}\right)}{2}\left(z-s_{n}\right)^{2}+\ldots
$$

Thus, for $0<\left|z-s_{n}\right|<\delta$,

$$
\begin{aligned}
F_{n}^{\prime}(z) & =\frac{A_{n}\left(1-z^{2}\right) P_{n}(z)}{\left(z-\bar{s}_{n}\right)^{2}\left(z-s_{n}\right)^{2}} \\
& =A_{n}\left[\frac{B_{-2}}{\left(z-s_{n}\right)^{2}}+\frac{B_{-1}}{\left(z-s_{n}\right)}+B_{0}+B_{1}\left(z-s_{n}\right)+\ldots\right] .
\end{aligned}
$$

Let $D=\left\{z:\left|z-s_{n}\right|<\delta\right\}-\left\{z: z=s_{n}+t e^{i \arg s_{n}}, 0 \leq t \leq \delta\right\}$. If $z_{0}=s_{n}+t e^{i \arg s_{n}},-\delta<t<0, z_{0}$ fixed, then for $z \in D$,

$$
F_{n}(z)-F_{n}\left(z_{0}\right)=\int_{z_{0}}^{z} F_{n}^{\prime}(\zeta) d \zeta
$$

where the path of integration is in $D$. Thus for $z$ in $D$,

$$
F_{n}(z)=A_{n}\left[\frac{d_{-1}}{z-s_{n}}+d \log \left(z-s_{n}\right)+q(z)\right]
$$


where $q(z)$ is analytic at $z=s_{n}$, and

$$
d_{-1}=\frac{1-s_{n}^{2}}{4-c_{n}^{2}} P_{n}\left(s_{n}\right) .
$$

Thus $\operatorname{Re} d_{-1}>0$. We take the branch of $\log$ such that for $z$ in $D$,

$$
\log \left(z-s_{n}\right)=\ln \left|z-s_{n}\right|+i \arg \left(z-s_{n}\right)
$$

where $\arg s_{n}<\arg \left(z-s_{n}\right)<\arg s_{n}+2 \pi$. Thus for $z$ in $D$,

$$
\begin{aligned}
\operatorname{Re} f_{n}(z)=\operatorname{Re} F_{n}(z)= & A_{n}\left[\operatorname{Re} \frac{d_{1}}{z-s_{n}}+(\operatorname{Re} d) \ln \left|z-s_{n}\right|\right. \\
& \left.-(\operatorname{Im} d) \arg \left(z-s_{n}\right)+\operatorname{Re} q(z)\right]
\end{aligned}
$$

We want to prove that $f_{n}(z)$ cannot have a finite cluster point at $z=s_{n}$.

Let $z_{j}=s_{n}+t_{j} e^{i \theta_{j}}$ be in $U \cap D$ with $t_{j}>0$ and $\lim t_{j}=0$ and such that

$$
\lim _{j \rightarrow \infty} \operatorname{Im}\left(\frac{z_{j}}{\left(1+c_{n} z_{j}+z_{j}^{2}\right)}\right)=l
$$

Straightforward computation gives

$$
\operatorname{Im}\left[\frac{z_{j}}{1+c_{n} z_{j}+z_{j}^{2}}\right]=\frac{-2\left(\operatorname{Im} s_{n}\right) \operatorname{Re}\left(s_{n} e^{-i \theta_{j}}\right)+t_{j} T_{j}}{t_{j}\left|2 i \operatorname{Im} s_{n}+t_{j} e^{i \theta_{j}}\right|^{2}}
$$

where $T_{j}$ is bounded. Because of (3.4), we must have

$$
\lim _{j \rightarrow \infty} \operatorname{Re}\left(s_{n} e^{-i \theta_{j}}\right)=0 .
$$

We now note that

$$
\begin{aligned}
d_{-1} e^{-i \theta_{j}} & =\frac{\left(1-s_{n}^{2}\right) e^{-i \theta_{j}} P_{n}\left(s_{n}\right)}{4-c_{n}^{2}}=\frac{\left(1 / s_{n}-s_{n}\right) s_{n} e^{-i \theta_{j}} P_{n}\left(s_{n}\right)}{4-c_{n}^{2}} \\
& =\frac{\left(\bar{s}_{n}-s_{n}\right) s_{n} e^{-i \theta_{j}} P_{n}\left(s_{n}\right)}{4-c_{n}^{2}}=\frac{-2 i\left(\operatorname{Im} s_{n}\right) s_{n} e^{-i \theta_{j}} P_{n}\left(s_{n}\right)}{4-c_{n}^{2}} .
\end{aligned}
$$

Thus,

$$
\begin{aligned}
\operatorname{Re}\left(d_{-1} e^{-i \theta_{j}}\right) & =\frac{2\left(\operatorname{Im} s_{n}\right) \operatorname{Im}\left(s_{n} e^{-i \theta_{j}} P_{n}\left(s_{n}\right)\right)}{4-c_{n}^{2}} \\
& =\frac{\operatorname{Im}\left(s_{n} e^{-i \theta_{j}} P_{n}\left(s_{n}\right)\right)}{\sqrt{4-c_{n}^{2}}} .
\end{aligned}
$$

Since $\lim _{j \rightarrow \infty} \operatorname{Re}\left(s_{n} e^{-i \theta_{j}}\right)=0$, it follows that the only possible accumulation points of $\left\{s_{n} e^{-i \theta_{j}}\right\}$ are $\pm i$. Thus the only possible accumulation points of $\left\{s_{n} e^{-i \theta_{j}} P_{n}\left(s_{n}\right)\right\}$ are $\pm i P_{n}\left(s_{n}\right)$. Moreover, $\operatorname{Im}\left( \pm i P_{n}\left(s_{n}\right)\right)= \pm \operatorname{Re} P_{n}\left(s_{n}\right) \neq 0$. Thus $\operatorname{Re}\left(d_{-1} e^{-i \theta_{j}}\right)$ is bounded away from 0 . 
It now follows that

$\left|\operatorname{Re} f_{n}\left(z_{j}\right)\right|$

$$
\begin{aligned}
& =\left|\operatorname{Re} F_{n}\left(z_{j}\right)\right| \\
& =A_{n}\left|\frac{\operatorname{Re}\left(d_{-1} e^{-i \theta_{j}}\right)}{t_{j}}+(\operatorname{Re} d) \ln \left(t_{j}\right)-(\operatorname{Im} d) \arg \left(t_{j} e^{-i \theta_{j}}\right)+\operatorname{Re} q\left(z_{j}\right)\right| \\
& =A_{n}\left|\frac{\operatorname{Re}\left(d_{-1} e^{-i \theta_{j}}\right)+(\operatorname{Re} d) t_{j} \ln \left(t_{j}\right)-t_{j}(\operatorname{Im} d) \arg \left(t_{j} e^{-i \theta_{j}}\right)}{t_{j}}+\operatorname{Re} q\left(z_{j}\right)\right|
\end{aligned}
$$

approaches $\infty$ as $j \rightarrow \infty$. Thus $f_{n}$ has no finite cluster points at $z=s_{n}$.

Similarly, $f_{n}$ has no finite cluster points at $z=\bar{s}_{n}$. At all other points of $|z|=1$, the finite cluster points of $f_{n}(z)$ are real. Since $f_{n}(U) \subset \Omega(a, b)$ and $\lim _{r \rightarrow-1^{+}} f_{n}(r)=a$ and $\lim _{r \rightarrow 1^{-}} f_{n}(r)=b$, it follows that $f_{n}(U)=\Omega(a, b)$.

Thus for each $n, f_{n}$ is a member of $S_{H}(U, \Omega(a, b))$. We know that the $P_{n}$ converge to $P$ uniformly on compact subsets of $U$. There exists a subsequence $c_{n_{k}}$ convergent to some $s$. But then (3.2) will be satisfied with $c$ replaced by $s$. Since the solution to (3.2) is unique, we must have $s=c$. Thus $f_{n_{k}}$ converges to $f$ uniformly on compact subsets of $U$. Therefore, $f$ is a member of $\overline{S_{H}(U, \Omega(a, b))}$ and $\mathcal{F}(a, b) \subset \overline{S_{H}(U, \Omega(a, b))}$. Since $\mathcal{F}(a, b)$ is closed and $S_{H}(U, \Omega(a, b)) \subset \mathcal{F}(a, b)$, we have $\overline{S_{H}(U, \Omega(a, b))} \subset \mathcal{F}(a, b)$. Thus $\mathcal{F}(a, b)=\overline{S_{H}(U, \Omega(a, b))}$.

4. The case $a=-b$. Referring to the proof of Lemma 1 , if $a=-b$ then

$$
T(0)=\int_{0}^{1} \frac{-4 b t}{\left(1+t^{2}\right)^{2}} d t<0 .
$$

Thus $c_{2}>0$. Moreover, since $S(-x)=-T(x)$, we have $c_{1}=-c_{2}$.

Since $S_{H}(U, \Omega(-b, b))$ are the only classes that contain odd functions, we will be interested in $f$ in $\mathcal{F}(-b, b)$ and $f$ odd.

Lemma 5. Let $f \in \mathcal{F}(-b, b)$ and be odd. If $f(z)=h(z)+\overline{g(z)}$, then both $h$ and $g$ are odd.

Pro of. Since $f(-z)=-f(z)$, we have $h(z)+\overline{g(z)}=-(h(-z)+\overline{g(-z)})$. Thus $h(z)+h(-z)=-\overline{g(z)+g(-z)}$. It follows that $h(z)+h(-z)$ and $\overline{h(z)+h(-z)}$ are both analytic in $U$. Thus $h(z)+h(-z)$ is constant. Since its value is 0 at $z=0$, we have $h(z)=-h(-z)$. Similarly, $g(z)$ is odd.

Lemma 6. If $f \in \mathcal{F}(-b, b)$ and $f$ is odd then in the representation (3.1), $P(z)$ is even and $c=0$. 
Proof. Let $h(z)=a_{1} z+a_{2} z^{2}+\ldots ;$ then

$$
h(z)=\frac{F(z)+i G(z)}{2}=\frac{a_{1}}{2}\left[\int_{0}^{z} \frac{\left(1-\zeta^{2}\right) P(\zeta)}{\left(1+c \zeta+\zeta^{2}\right)^{2}} d \zeta+\frac{z}{1+c z+z^{2}}\right]
$$

where $c$ and $P$ satisfy $(3.2)$. Since $\left(1-z^{2}\right) /\left(1+c z+z^{2}\right)^{2}=\left(z /\left(1+c z+z^{2}\right)\right)^{\prime}$, this can be written as

$$
h(z)=\frac{a_{1}}{2} \int_{0}^{z} \frac{\left(1-\zeta^{2}\right)(P(\zeta)+1)}{\left(1+c \zeta+\zeta^{2}\right)^{2}} d \zeta
$$

By Lemma $5, h(z)=-h(-z)$. Thus,

$$
\int_{0}^{z} \frac{\left(1-\zeta^{2}\right)(P(\zeta)+1)}{\left(1+c \zeta+\zeta^{2}\right)^{2}} d \zeta=-\int_{0}^{-z} \frac{\left(1-\zeta^{2}\right)(P(\zeta)+1)}{\left(1+c \zeta+\zeta^{2}\right)^{2}} d \zeta
$$

Let $z=r, 0<r<1$; then

$$
\int_{0}^{r} \frac{\left(1-t^{2}\right)(P(t)+1)}{\left(1+c t+t^{2}\right)^{2}} d t=\int_{0}^{r} \frac{\left(1-t^{2}\right)(P(-t)+1)}{\left(1-c t+t^{2}\right)^{2}} d t .
$$

Taking real parts, we get

$$
\int_{0}^{r} \frac{\left(1-t^{2}\right)(\operatorname{Re} P(t)+1)}{\left(1+c t+t^{2}\right)^{2}} d t=\int_{0}^{1} \frac{\left(1-t^{2}\right)(\operatorname{Re} P(-t)+1)}{\left(1-c t+t^{2}\right)^{2}} d t .
$$

Letting $r \rightarrow 1$, since $-2<-c_{2} \leq c \leq c_{2}<2$, we obtain

$$
\int_{0}^{1} \frac{\left(1-t^{2}\right)(\operatorname{Re} P(t)+1)}{\left(1+c t+t^{2}\right)^{2}} d t=\int_{0}^{1} \frac{\left(1-t^{2}\right)(\operatorname{Re} P(-t)+1)}{\left(1-c t+t^{2}\right)^{2}} d t .
$$

But (3.2) with $a=-b$ gives

$$
\int_{0}^{1} \frac{\left(1-t^{2}\right) \operatorname{Re} P(-t)}{\left(1-c t+t^{2}\right)^{2}} d t=\int_{0}^{1} \frac{\left(1-t^{2}\right) \operatorname{Re} P(t)}{\left(1+c t+t^{2}\right)^{2}} d t
$$

Equalities (4.1) and (4.2) imply

$$
\int_{0}^{1} \frac{1-t^{2}}{\left(1+c t+t^{2}\right)^{2}} d t=\int_{0}^{1} \frac{1-t^{2}}{\left(1-c t+t^{2}\right)^{2}} d t .
$$

Thus $1 /(2+c)=1 /(2-c)$. Hence $c=0$.

We now have

$$
h(z)=\frac{a_{1}}{2}\left[\int_{0}^{z} \frac{\left(1-\zeta^{2}\right) P(\zeta)}{\left(1+\zeta^{2}\right)^{2}} d \zeta+\frac{z}{1+z^{2}}\right]
$$


and $h(z)$ is odd. Thus

$$
q(z)=\int_{0}^{z} \frac{\left(1-\zeta^{2}\right) P(\zeta)}{\left(1+\zeta^{2}\right)^{2}} d \zeta
$$

is odd. Hence $q^{\prime}(z)=\left(1-z^{2}\right) P(z) /\left(1+z^{2}\right)^{2}$ is even and thus $P(z)$ is even.

Lemma 7. Let $f \in \mathcal{F}(-b, b)$ with representation (3.1). If $P(z)$ is even, then $c=0$ and $f$ is odd.

P r o of. If $P(z)$ is even, then $Q(x)$ defined by (2.3), with $a=-b$, satisfies

$$
Q(0)=-\int_{0}^{1} \frac{\left(1-t^{2}\right) \operatorname{Re} P(t)}{\left(1+t^{2}\right)^{2}} d t+\int_{0}^{1} \frac{\left(1-t^{2}\right) \operatorname{Re} P(-t)}{\left(1+t^{2}\right)^{2}} d t=0 .
$$

But the $c$ given in Lemma 2 is unique. Thus $c=0$. Therefore

$$
f(z)=a_{1}\left[\operatorname{Re} \int_{0}^{z} \frac{\left(1-\zeta^{2}\right) P(\zeta)}{\left(1+\zeta^{2}\right)^{2}} d \zeta+i \operatorname{Im} \frac{z}{\left(1+z^{2}\right)}\right]
$$

and since $P(z)$ is even, it is easily checked that $f(-z)=-f(z)$.

We now let

$$
G(-b, b)=\{f \in \mathcal{F}(-b, b): f \text { is odd }\} .
$$

If $f \in G(-b, b)$, then $f$ has the representation (4.3) with $P(z)$ in $\mathcal{P}$ and $P(z)$ even. Also,

$$
a_{1}=b / \int_{0}^{1} \frac{\left(1-t^{2}\right) \operatorname{Re} P(t)}{\left(1+t^{2}\right)^{2}} d t
$$

We now easily obtain

Theorem 4. If $f \in G(-b, b)$, then

$$
\frac{4 b}{\pi} \leq a_{1} \leq \frac{8 b}{\pi}
$$

and the inequalities are sharp.

Proof. Since $P \in \mathcal{P}$ and $P$ is even, $\left(1-|z|^{2}\right) /\left(1+|z|^{2}\right) \leq \operatorname{Re} P(z) \leq$ $\left(1+|z|^{2}\right) /\left(1-|z|^{2}\right)$. Thus

$$
\frac{\pi}{8}=\int_{0}^{1} \frac{\left(1-t^{2}\right)^{2}}{\left(1+t^{2}\right)^{3}} d t \leq \int_{0}^{1} \frac{\left(1-t^{2}\right) \operatorname{Re} P(t)}{\left(1+t^{2}\right)^{2}} d t \leq \int_{0}^{1} \frac{d t}{1+t^{2}}=\frac{\pi}{4}
$$

and the result follows from (4.4). Equality is attained on the right side of (4.5) when $P(z)=\left(1-z^{2}\right) /\left(1+z^{2}\right)$ and on the left side when $P(z)=$ $\left(1+z^{2}\right) /\left(1-z^{2}\right)$. The corresponding extremal functions are

$$
f_{1}(z)=\frac{8 b}{\pi}\left[\operatorname{Re}\left[\frac{z\left(1-z^{2}\right)}{2\left(1+z^{2}\right)^{2}}+\frac{1}{2} \arctan z\right]+i \operatorname{Im} \frac{z}{1+z^{2}}\right]
$$


and

$$
f_{2}(z)=\frac{4 b}{\pi}\left[\operatorname{Re}(\arctan (z))+i \operatorname{Im} \frac{z}{1+z^{2}}\right] .
$$

We find in Section 5 that $f_{1}(z)$ is actually a member of $S_{H}(U, \Omega(-b, b))$. Thus the right side of (4.5) is sharp for odd functions in $S_{H}(U, \Omega(-b, b))$.

TheOrem 5. Let $f(z)=h(z)+\overline{g(z)}$ be in $G(-b, b)$ and suppose

$$
h(z)=\sum_{n=0}^{\infty} a_{2 n+1} z^{2 n+1} \quad \text { and } \quad g(z)=\sum_{n=1}^{\infty} b_{2 n+1} z^{2 n+1} .
$$

Then

$$
\begin{aligned}
& \left|a_{2 n+1}\right| \leq \frac{(n+1)^{2}}{2 n+1}\left|a_{1}\right|, \quad n=0,1,2, \ldots, \\
& \left|b_{2 n+1}\right| \leq \frac{n^{2}}{2 n+1}\left|a_{1}\right|, \quad n=1,2, \ldots,
\end{aligned}
$$

and

$$
\left|a_{2 n+1}-b_{2 n+1}\right|=\left|a_{1}\right|
$$

and the inequalities are sharp in $S_{H}(U, \Omega(-b, b))$.

Proof. We have

$$
h(z)=\frac{a_{1}}{2}\left[\int_{0}^{z} \frac{\left(1-\zeta^{2}\right) P(\zeta)}{\left(1+\zeta^{2}\right)^{2}} d \zeta+\frac{z}{1+z^{2}}\right]
$$

where $P(z)$ is in $\mathcal{P}$ and is even. Let $P(z)=1+\sum_{n=1}^{\infty} p_{2 n} z^{2 n}$; then for $|z|<1$,

$$
\frac{1-z^{2}}{\left(1+z^{2}\right)^{2}} P(z)=1+\sum_{n=1}^{\infty} d_{2 n} z^{2 n}
$$

where

$$
d_{2 n}=\sum_{k=0}^{n}(-1)^{k}(2 k+1) p_{2(n-k)} \quad \text { and } \quad p_{0}=1 .
$$

Then (4.11) gives

$$
\begin{aligned}
\frac{2 a_{2 n+1}}{a_{1}} & =\frac{1}{2 n+1} \sum_{k=0}^{n}(-1)^{k}(2 k+1) p_{2(n-k)}+(-1)^{n} \\
& =\frac{1}{2 n+1} \sum_{k=0}^{n-1}(-1)^{k}(2 k+1) p_{2(n-k)}+2(-1)^{n} .
\end{aligned}
$$


Since $\left|p_{n}\right| \leq 2$ for all $n$, we have

$$
\frac{2\left|a_{2 n+1}\right|}{\left|a_{1}\right|} \leq \frac{2}{2 n+1} \sum_{k=0}^{n-1}(2 k+1)+2=\frac{2 n^{2}}{2 n+1}+2=\frac{2(n+1)^{2}}{2 n+1},
$$

giving (4.8).

To see the sharpness, let $P(z)=\left(1-z^{2}\right) /\left(1+z^{2}\right)$. With this choice of $P$, we have $p_{2 n}=2(-1)^{n}$ and from (4.12),

$$
\begin{aligned}
\frac{2 a_{2 n+1}}{a_{1}} & =\frac{1}{2 n+1} \sum_{k=0}^{n-1}(-1)^{k}(2 k+1)(-1)^{n-k} \cdot 2+2(-1)^{n} \\
& =(-1)^{n}\left[\frac{2}{(2 n+1)} \sum_{k=0}^{n-1}(2 k+1)+2\right]=\frac{2(-1)^{n}(n+1)^{2}}{2 n+1},
\end{aligned}
$$

giving equality in (4.8). The extremal function is the $f_{1}(z)$ given in (4.6).

Next we have

$$
g(z)=\frac{a_{1}}{2}\left[\int_{0}^{z} \frac{\left(1-\zeta^{2}\right) P(\zeta)}{\left(1+\zeta^{2}\right)^{2}} d \zeta-\frac{z}{1+z^{2}}\right]
$$

If $g(z)=\sum_{n=1}^{\infty} b_{2 n+1} z^{2 n+1}$, then

$$
\frac{2 b_{2 n+2}}{a_{1}}=\frac{1}{2 n+1} \sum_{k=0}^{n-1}(-1)^{k}(2 k+1) p_{2(n-k)} .
$$

Thus

$$
\frac{2\left|b_{2 n+1}\right|}{\left|a_{1}\right|} \leq \frac{2}{2 n+1} \sum_{k=0}^{n-1}(2 k+1)=\frac{2 n^{2}}{2 n+1},
$$

giving (4.9). Equality again occurs when $P(z)=\left(1-z^{2}\right) /\left(1+z^{2}\right)$ and $f_{1}(z)$ is given in (4.6).

Finally, from (4.10) and (4.11),

$$
\left|a_{2 n+1}-b_{2 n+1}\right|=\left|(-1)^{n} a_{1}\right|=\left|a_{1}\right| .
$$

We remark that the inequalities involved are actually sharp for odd functions in $S_{H}(U, \Omega(-b, b))$ since $f_{1} \in S_{H}(U, \Omega(-b, b))$.

TheOREM 6. Let $f(z)=h(z)+\overline{g(z)}$ be a member of $G(-b, b)$. Then for $|z|=r<1$,

$$
\frac{\left|a_{1}\right|\left(1-r^{2}\right)}{\left(1+r^{2}\right)^{3}} \leq\left|f_{z}(z)\right| \leq \frac{\left|a_{1}\right|\left(1+r^{2}\right)}{\left(1-r^{2}\right)^{3}}
$$

and the inequalities are sharp. 
Pr o of. We have

$$
h(z)=\frac{a_{1}}{2}\left[\int_{0}^{z} \frac{\left(1-\zeta^{2}\right) P(\zeta)}{\left(1+\zeta^{2}\right)^{2}} d \zeta+\frac{z}{1+z^{2}}\right] .
$$

Thus,

$$
f_{z}=h^{\prime}(z)=\frac{a_{1}\left(1-z^{2}\right)}{2\left(1+z^{2}\right)^{2}}(P(z)+1) .
$$

Since $P(z)$ is in $\mathcal{P}$ and is even, we can write $P(z)=(1-w(z)) /(1+w(z))$ where $w(z)=d_{2} z^{2}+\ldots$ is analytic in $U$ and $|w(z)| \leq|z|^{2}$ for $z$ in $U$. Thus $P(z)+1=2 /(1+w(z))$. Hence

$$
\frac{2}{1+r^{2}} \leq \frac{2}{1+|w(z)|} \leq|P(z)+1| \leq \frac{2}{1-|w(z)|} \leq \frac{2}{1-r^{2}} .
$$

Using (4.10) and (4.15) we obtain the inequalities (4.14). Equality on the right side of (4.14) is attained by $f_{1}(z)$ at $z= \pm i r$ and equality on the left side of (4.14) is attained by $f_{1}(z)$ when $z= \pm r$.

5. The extremal functions. We now verify that the extremal function $f_{1}(z)$ given by (4.6) is actually a member of $S_{H}(U, \Omega(-b, b))$, while the function $f_{2}(z)$ given by (4.7) maps $U$ into the strip $\{z:-b<\operatorname{Re} z<b\}$ and hence is a member of $G(-b, b)-S_{H}(U, \Omega(-b, b))$.

To see this we first prove that $f_{1}(z)$ has no non-real finite cluster points at $z=i$. Let $z_{j}=i+t_{j} e^{i \theta_{j}}$ be such that $0<t_{j}, \pi<\theta_{j}<2 \pi,\left|z_{j}\right|<1$, and $\lim _{j \rightarrow \infty} \operatorname{Im}\left(z_{j} /\left(1+z_{j}^{2}\right)\right)=l \neq 0$. Necessarily $l>0$. A brief computation gives

$$
A_{j}=\operatorname{Im}\left(\frac{z_{j}}{1+z_{j}^{2}}\right)=\frac{-\left(t_{j}+2 \sin \theta_{j}\right)\left(1+t_{j} \sin \theta_{j}\right)}{t_{j}\left|z_{j}+i\right|^{2}} .
$$

Thus $-\left(t_{j}+2 \sin \theta_{j}\right)\left(1+t_{j} \sin \theta_{j}\right)=t_{j}\left|z_{j}+i\right|^{2} A_{j}=t_{j} B_{j}$ where $\lim B_{j}=$ $4 l>0$. Hence

$$
-2 \sin \theta_{j}\left[1+t_{j} \sin \theta_{j}\right]=t_{j} B_{j}+t_{j}\left[1+t_{j} \sin \theta_{j}\right]=t_{j} c_{j},
$$

where $\lim c_{j}=4 l+1$. Therefore

$$
\sin \theta_{j}=\frac{t_{j} c_{j}}{-2\left(1+t_{j} \sin \theta_{j}\right)}=t_{j} D_{j}
$$

where $\lim D_{j}=-(4 l+1) / 2$. In particular, $\lim \sin \theta_{j}=0$, so $\lim \left|\cos \theta_{j}\right|=1$. Let

$$
T(z)=\frac{z\left(1-z^{2}\right)}{(z-i)^{2}(z+i)^{2}}
$$

then in a neighborhood of $z=i$,

$$
T(z)=\frac{-i}{2(z-i)^{2}}-\frac{1}{2(z-i)}+q(z)
$$


where $q(z)$ is analytic at $z=i$. Further,

$$
T\left(z_{j}\right)=\frac{-i e^{-i 2 \theta_{j}}}{2 t_{j}^{2}}-\frac{e^{-i \theta_{j}}}{2 t_{j}}+q\left(z_{j}\right) .
$$

Using (5.1), we can write

$$
\begin{aligned}
\operatorname{Re} T\left(z_{j}\right) & =\frac{\sin \theta_{j} \cos \theta_{j}}{t_{j}^{2}}-\frac{\cos \theta_{j}}{2 t_{j}}+\operatorname{Re} q\left(z_{j}\right) \\
& =\frac{-D_{j} \cos \theta_{j}}{t_{j}}-\frac{\cos \theta_{j}}{2 t_{j}}+\operatorname{Re} q\left(z_{j}\right)=\frac{-\cos \theta_{j}\left(2 D_{j}+1\right)}{2 t_{j}}+\operatorname{Re} q\left(z_{j}\right) .
\end{aligned}
$$

Since $\lim \left(2 D_{j}+1\right)=-4 l \neq 0$ and $\lim \left|\cos \theta_{j}\right|=1$ it follows that $\lim \left|\operatorname{Re} T\left(z_{j}\right)\right|$ $=\infty$ and hence $\lim \left|\operatorname{Re} f_{1}\left(z_{j}\right)\right|=\infty$. Thus $f_{1}$ has only real cluster points at $z=i$. Since $f_{1}(z)$ is odd, it has only real cluster points at $z=-i$ as well. If $z_{0} \neq \pm i$ and $\left|z_{0}\right|=1$, then $\lim _{z \rightarrow z_{0}} f_{1}(z)= \pm b$. Since $f_{1}(U) \subset \Omega(-b, b)$ and since the interval $(-b, b)$ is covered by $f_{1}(U)$, it follows that $f_{1}(U)=$ $\Omega(-b, b)$. Thus $f_{1}$ is a member of $S_{H}(U, \Omega(-b, b))$.

We now prove that $f_{2}(U)=\{z:-b<\operatorname{Re} z<b\}$ where $f_{2}(z)$ is given by (4.7). We have

$$
\operatorname{Re} f_{2}(z)=\frac{4 b}{\pi} \operatorname{Re}(\arctan z)=\frac{4 b}{\pi} \operatorname{Re}\left(\frac{i}{2} \log \frac{1-i z}{1+i z}\right)=\frac{-2 b}{\pi} \arg \left(\frac{1-i z}{1+i z}\right) .
$$

Since $\operatorname{Re}[(1-i z) /(1+i z)]>0$, it follows that

$$
\left|\operatorname{Re} f_{2}(z)\right|=\frac{2 b}{\pi}\left|\arg \frac{1-i z}{1+i z}\right|<\frac{2 b}{\pi} \cdot \frac{\pi}{2}=b .
$$

We claim that the cluster points of $f_{1}(z)$ at $z= \pm i$ form the two lines $\operatorname{Re} z= \pm b$. To see this, let $l>0$. We can choose a sequence $z_{j}=i+t_{j} e^{-i \theta_{j}}$ with $\pi<\theta_{j}<2 \pi, t_{j}>0$ and $\lim t_{j}=0$, such that

$$
\lim _{j \rightarrow \infty} \operatorname{Im} \frac{z_{j}}{1+z_{j}^{2}}=l \text {. }
$$

As in the previous example, $\lim \sin \theta_{j}=0$ and $\lim \left|\cos \theta_{j}\right|=1$. We have

$$
\operatorname{Re} f_{2}\left(z_{j}\right)=-\frac{2 b}{\pi} \arg \left(\frac{1-i z_{j}}{1+i z_{j}}\right)
$$

Moreover,

$$
\tan \left[\arg \left(\frac{1-i z_{j}}{1+i z_{j}}\right)\right]=\frac{-2 \operatorname{Re} z_{j}}{1-\left|z_{j}\right|^{2}}=\frac{-2 t_{j} \cos \theta_{j}}{-2 t_{j} \sin \theta_{j}-t_{j}^{2}}=\frac{2 \cos \theta_{j}}{2 \sin \theta_{j}+t_{j}} .
$$

Making use of computations from the last example, we get

$$
\tan \left[\arg \left(\frac{1-i z_{j}}{1+i z_{j}}\right)\right]=\frac{2 \cos \theta_{j}}{2 t_{j} D_{j}+t_{j}}=\frac{2 \cos \theta_{j}}{t_{j}\left(2 D_{j}+1\right)}
$$

where $\lim 2 D_{j}+1=-4 l<0$. 
If $\theta_{j}$ is chosen so that $\lim \theta_{j}=\pi$ then $\tan \left(\arg \left(\left(1-i z_{j}\right) /\left(1+i z_{j}\right)\right)\right)$ tends to $\infty$ and $\arg \left(\left(1-i z_{j}\right) /\left(1+i z_{j}\right)\right)$ tends to $\pi / 2$, and thus $\operatorname{Re} f_{2}\left(z_{j}\right)$ tends to $-b$. Hence $-b+i l, l>0$, is a cluster point. If $\theta_{j}$ is chosen so that $\lim \theta_{j}=2 \pi$, then we see that $b+i l, l>0$, is a cluster point. Since $f_{2}$ is odd, it follows that $\pm b+i l, l<0$, are cluster points at $z=-i$. It now follows that $f_{2}(U)=\{z:-b<\operatorname{Re}(z)<b\}$.

\section{References}

[1] Y. Abu-Muhanna and G. Schober, Harmonic mappings onto convex domains, Canad. J. Math. 39 (1987), 1489-1530.

[2] J. A. Cima and A. E. Livingston, Integral smoothness properties of some harmonic mappings, Complex Variables Theory Appl. 11 (1989), 95-110.

[3] - - - Nonbasic harmonic maps onto convex wedges, Colloq. Math. 66 (1993), 9-22.

[4] J. Clunie and T. Sheil-Small, Harmonic univalent functions, Ann. Acad. Sci. Fenn. Ser. A I Math. 9 (1984), 3-25.

[5] W. Hengartner and G. Schober, Univalent harmonic functions, Trans. Amer. Math. Soc. 299 (1987), 1-31.

[6] -, - Curvature estimates for some minimal surfaces, in: Complex Analysis, J. Hersch and A. Huber (eds.), Birkhäuser, 1988, 87-100.

[7] A. E. Livingston, Univalent harmonic mappings, Ann. Polon. Math. 57 (1992), $57-70$.

Department of Mathematics

University of Delaware

Newark, Delaware 19716

U.S.A.

E-mail: livingst@math.udel.edu 